\title{
ESQUIZOFRENIA E ORDEM DE NASCIMENTO
}

\author{
PAULO CESAR SANDLER * \\ ESTER HADASSA SANDLER **
}

A possivel influência de fatores anıbientais na etipatogenia das doenças mentais tem sido a preocupação do corpo de doutrina ao qual se convencionou chamar "Psiquiatria Social", que muitas vezes tem mereciclo conotações de um verdadeiro movimento ${ }^{34,37}$. Deve-se notar que a Psiquiatria Social ainda não tem modelos teóricos $1,21,34,3 i, 41,49$ existindo, no entanto, métodos científicos emprestados de outras ciências 37,49 que se mostram úteis quando aplicados na investigação das variáveis ambientais que possam estar desempenhando algum papel na gênese, manutenção e reabilitação das doenças psiquiátricas. $O$ método mais bem firmado é, sem dúvida, o epidemiológico ${ }^{0,30,34}$ que, mesmo não pretendendo fornecer respostas finais ou deslindar por completo as redes multifatoriais de causalidade, pode emprestar validade cientifica a conclusões sobre determinadas facetas dos quadros etiológicos. A própria Organização Mundial de Saúde considera como valiosa a pesquisa orientada estatisticamente, tendo feito várias recomendações neste sentido 20 .

Dentro das variáveis que a Psiquiatria Social enfoca, têm merecido especial atenção as micro-sociais, ou seja, o micro-cosmo do doente mental 36,37. Considerando-se estas variáveis, os dados familiares são objeto de estudo cada vez mais acurado, quer seja por meio de modelos interacionais 4,48 quer seja pelo uso do próprio método epidemiológico ${ }^{9}$. Assim, a quantificação de dados familiares abjetivos tem ocupado grande parte da literatura. $O$ dado mais bem estudado tem sido a ordem de nascimento dos pacientes, ou seja, a sua posição dentro da prole, e sua possível ocorrência concomitante à prevalência 0,24 das várias doenças psiquiátricas.

Assim, uma hipotética relação entre a doença esquizofrênica e a ordem de nascimento tem sido continuamente testada pelos investigadores. Esta relação oferece uma série de hipóteses subsequentes de trabalho, a serem verificadas não só pelo método epidemiológico, mas por outros que ofereçam alguma explicação a respeito das associações porventura encontradas.

O presente estudo tem por objetivo verificar esta possível associação. cuidando de uma patologia que constitui um dos maiores problemas teóricos e

Trabalho realizado sob bolsa da Fundação de Amparo à Pesquisa do Estado de São Paulo, proc. 70/1183 e 72/299; orientador, Dr. Euclides Ayres de Castilho. do Departamento de Medicina Preventiva da Faculdade de Medicina da Universidade de São Paulo: *Psiquiatra na Faculdade de Saúde Pública da Universidade de São Paulo, médico assistente do Instituto Aché; **médica-residente de Psiquiatria na Faculdade de Medicina da Universidade de São Paulo. 
práticos para o psiquiatra 19: a doença esquizofrênica. Referimo-nos ao grupo das esquizofrenias, como uma entidade nosológica cuja descrição segue os padrões de Bleuler e de Kraepelin, pois esta é a orientação diagnóstica dos três hospitais de onde provêm os doentes ${ }^{5,8}$.

Em relação ao tema específico, embora o número de estudos na literatura passe de três dezenas, não há conclusão definitiva alguma, sendo muito alta a taxa de discrepância nos resultados, como veremos adiante. Esta discrepância é de tal ordem que alguns pesquisadores chegaram a propor que não mais se estudasse o problema ?. Estudos desta natureza ainda não foram realizados em nosso meio.

\section{METODOS E MATERIAL}

O método básico para poder analisar efeitos de ordem de nascimento e a prevalência de doenças foi proposto no início do século, por Greenwood-Yule (cit. em McMahon e col. 24). O método de Greenwood-Yule é um instrumento estatístico que formula hipótese inicial e lança mão de uma construção estatística para o estabelecimento de um grupo controle, baseado nesta hipótese, com o intuito de testar o efeito da ordem de nascimento.

Sua hipótese nula 9,39, é de que não existe associação estatística entre a ordem de nascimento e a prevalência da doença em questão. Como consequência, a probabilidade de um indivíduo de uma determinada prole, que seja afetado pela doença, nascer em qualquer uma das posições possíveis, é sempre a mesma. Em outras palavras, a distribuição das frequências de ordem de nascimento é randomizada.

Assim, toma-se um grupo controle de acordo com tsta hipótese nula; este grupo controle é comparado por meio de um teste estatístico com o grupo de doentes obtidos (casuistica de dados hospitalares): distribuem-se as frequências da casuística em uma tabela de ordem de nascimento, conforme o tamanho da prole definida como o número de filhos. Em outras palavras, dada uma prole de $N$ indivíduos, para uma ordem de nascimento $K$, a probabilidade de termos individuos afetados será dada por $\mathbf{N} / \mathbf{K}$.

O método de Greenwood-Yule, apesar de ser o mais antigo, tem resistido a criticas14,29 e ainda é considerado como bom, sendo pelo menos tão válido quanto outros propostos recentemente 33,40 . Ele foi largamente usado pelos pesquisadores, desde os primeiros até os mais recentes 2,22. Entretanto, o método original de GreenwoodYule fazia uso do qui quadrado (x2) clássico como teste de aderência para comparação das frequências das ordens de nascimento. Consideramos que é bem possível que seja esta a causa da alta taxa de discordência nos resultados dos vários autores, pois está demonstrado que este teste não é suficientemente sensivel para testar efeitos de ordem de nascimentos, cuja distribuição não segue a curva do qui quadrado 13,20.

Max Halperin, matemático norte-americano, propôs em 1953 um qui quadrado modificado que envolve a inversão da matriz quadrática que é obtida quando se dispõe os dados segundo o método de Greenwood-Yule; neste mesmo estudo ele nota a inadequação do qui quadrả̉o clássico 13. Dado o fato que o modelo matemático desenvolvido por Halperin é complexo, envolvendo certas dificuldades técnicas em sua aplicação, em 1963 o mesmo autor, juntamente com Mantel, propuseram uma simplificação do método 23, na qual a variança e a co-variança da matriz mencionada são

Nota dos autores - Deixamos expresso o nosso agradecimento ao Dr. Cláudio Luis Lucarelli por sua ajuda na coleta de dados e aos Drs. Mario Yahn, Waldemar Cardoso. Eugenio Mariz de Oliveira Netto e H. Carvalho, diretores clínicos dos hospitais de onde foram coletados os dados (Instituto Aché, Sanatório Bela Vista e Hospital das Clínicas da Faculdade de Medicina da Universidade de São Paulo). 
obtídas sem a necessidade de sua Inversão, evitando-se assim o uso de computação eletrônica para os cálculos aritméticos. Vale notar que nem o método original nem a simplificação foram usadas, até o momento.

Assim, o método utilizado no presente estudo os o de Greenwood-Yule e, o teste de aderência, o qui quadrado modificado por Halperin. Fixamos o nível de significância em 0.01 .

Obtivemos os dados de ordem de nascimento de 1016 pacientes que provinham de três hospitais da área metropolitana de São Paulo*. Os diagnósticos se baseavam nos registros hospitalares, sendo que a orientação diagnóstica era semelhante nos très hospitais; na verdade, alguns dos médicos que fizeram os diagnósticos trabalhavam simultaneamente em dois deles. Os casos duvidosos foram sumariamente rejeitados. Todos os pacientes eram de primeiras admissóes, tendo silo coberto um período de 12 anos (de 1960 a 1972); 98 pacientes vieram do hospital A, 297 do hospital B e 619 do hospital C. A seleção de três hospitais diferentes se deu pelo fato de que o fator classe social modifica a prevalência da esquizofrenia na população geral 17. Na tentativa de controlar qualitativamente esta variável, os hospitais foram escolhidos de maneira a se incluir uma larga gama de estratos sociais. Pode-se observar que o número de pacientes obtidos em cada hospital é proporcional, de maneira aproximada, à prevalência da esquizofrenia nos vários estratos sociais.

Só foram aproveitados os pacientes com diagnóstico de certeza que tivessem mais de 15 anos de idade, para que não trabalhássemos com proles incompletas, o que produziria maior peso nos primeiros nascidos 24,2i. Os irmãos mortos na primeira infancia não foram computados, pois, ao se estudar um fator presumivelmente ambiental, estes irmãos mortos precocemente não formaram o ambiente de vida do paciente: Consultaram-se cerca de 2500 prontuários; os 1484 rejeltados não continham o registro da ordem de nascomento ou não respeitavam alguns dos pontos mencionados acima.

\section{RESULTADOS}

Os 1016 pacientes dividiam-se, quanto ao sexo, em 642 homens e 374 mulheres. Os gêmeos foram tratados segundo McMahon e col. 24. A tabela 1 mostra a distribuição etária, sendo a idade mídia de nossa casuística de 22 anos.

A tabela 2 mostra os valores observados das frequências das ordens de nascimento dos 1016 pacientes; a tabela 3 mostra as frequencias esperadas segundo 0 método de Greenwood-Yule.

As frequências observadas e esperadas das ordens de nascimento, para serem comparadas pelo teste estatístico de Halperin, figuram na tabela 4. O valor da estatís

\begin{tabular}{lc||cc}
\hline $\begin{array}{l}\text { Intervalo } \\
\text { de idade }\end{array}$ & Frequências & $\begin{array}{l}\text { Intervalo } \\
\text { de idade }\end{array}$ & Frequências \\
\hline $15-20$ & $313(30.80 \%)$ & $41-45$ & $45(4.42 \%)$ \\
$21-25$ & $260(25.59 \%)$ & $46-50$ & $26(2.55 \%)$ \\
$26-30$ & $182(17.91 \%)$ & $51-55$ & $8(0.78 \%)$ \\
$31-35$ & $110(10.82 \%)$ & $56 \mathrm{e}+$ & $10(0.98 \%)$ \\
$36-40$ & $62(6.10 \%)$ & & $56 \%$ \\
\hline
\end{tabular}

Tabela 1 - Distribuição agrupada das idxdes de 1016 esquizofrênicos brasileiros

- Hospital A é um nosocómio de médio porte, que recebe pacientes dos estratos sociais mais ricos, cuja internação é custea da por iniciativa privada: o Hospital B recebe pacientes de nível económico médio, mantendo convênio com entidade previdenciária estatal; o Hospital C é uma clinica estadual, funcionando anexa a uma universidade, recebendo pacientes dos cstratos sociais mais pobres. 


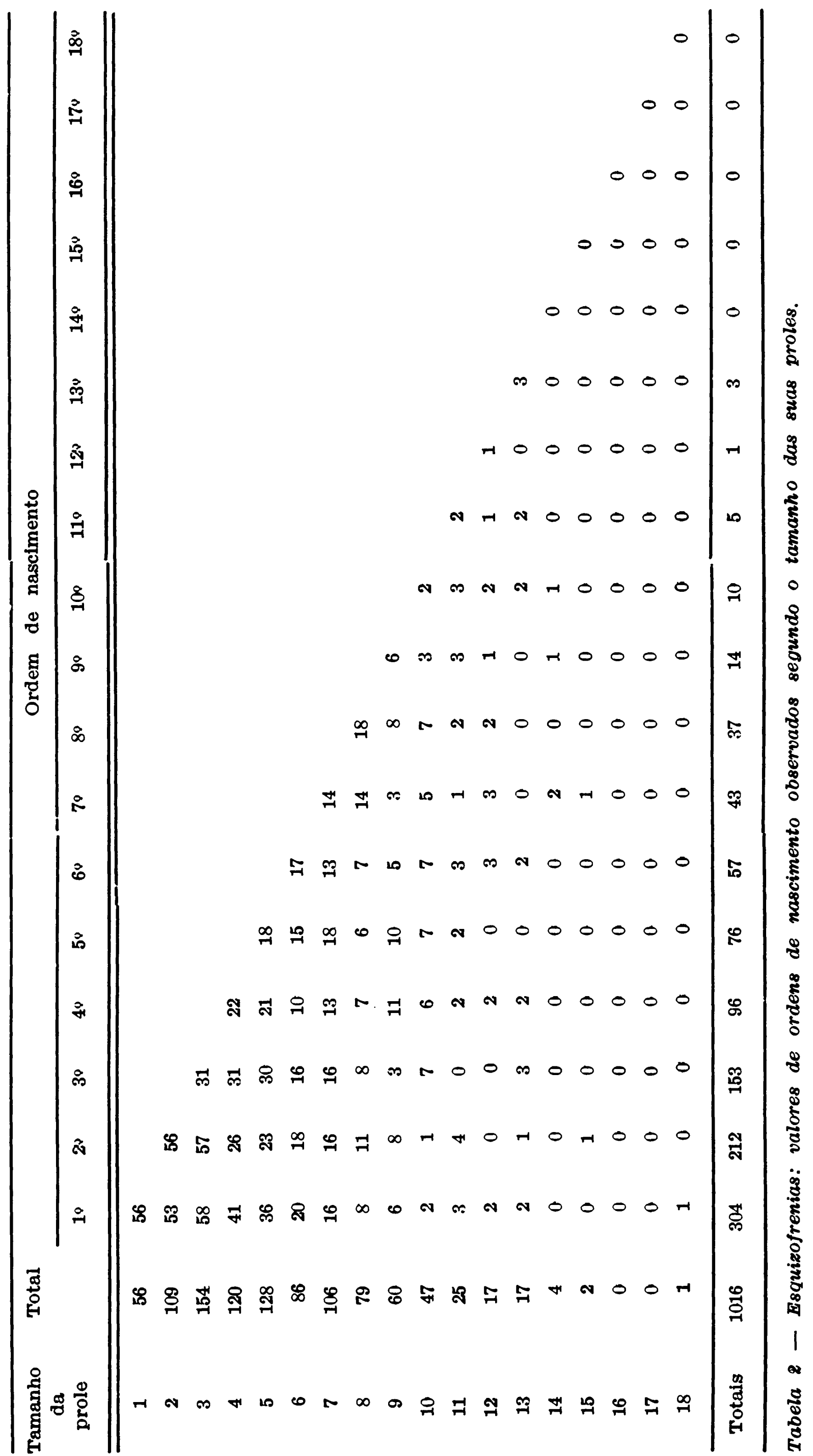




\begin{tabular}{ccc}
\hline $\begin{array}{c}\text { Or..em de } \\
\text { nascimento }\end{array}$ & Frequencias \\
\cline { 2 - 3 } & observado & csperado \\
\hline 1 & 304 & 273,57 \\
2 & 212 & 217,57 \\
3 & 153 & 163,07 \\
4 & 96 & 111,74 \\
5 & 76 & 81,74 \\
6 & 57 & 56,14 \\
7 & 43 & 11,81 \\
8 & 37 & 26,67 \\
9 & 33 & 38,1 \\
\hline Totais & 1016 & 1016,41 \\
\hline
\end{tabular}

Tabela 4 - Frequencias observadas e esperadas das ordens de nascimento de 1016 esquizofrericos brasileiros, segundo o metodo de GreenwoodYulc. $O$ valor do $x$ modificado é obtido pelo método de Halperin: $x 2$ modificado $=22.96 .3 ; 8 \mathrm{gl} ; \mathrm{p}<0.01$.

tica de Halperin obtido fol de 22.953, para 8 graus de liberdade, significativo ao nivel de 1\%. Isto nos permite afirmar que existe uma associaçåo estatisticamente significativa entre esquizofrenia e ordem de nascimento, em nossa casuistica.

As tabelas 5 e 7 mostram as frequências das ordens de nascimento observadas para os homens e mulheres, e as tabelas 6 e 8 , as frequências esperadas, também para os homens e mulheres, respectivamente, segundo o métoło de Greenwood-Yule. Os valores do qui quadrado modificado, que aparecem na tabela 9, nos mostram que existe influência do sexo na associação positiva encontrada na análise dos dados globais. Pode-se ver que a associação persiste entre homens esquizofrênicos (qui quadrado modificado $=22,83,8$ g.l.) mas ela não é encontrada entre as mulheres (qui quadrado modificado $=9.543,8$ g.1., năo significativo), ou seja, a associaçăo encontrada na casuistica global ó devida aos homens esquizofrénicos. Deve-sé notar que, dado o fato que foram considerados todos os tamanhos de familia, uma influência postulada do tamanho da prole permanece controlada.

A associaçăo encontrada, nos homens diagnosticados como esquizofrênicos, se dá, conforme se verifica na tabela 9 , em direçăo aos primeiros e últimos nascidos. De maneira sintética, podemos sumarizar js nossos resultados: foi encontrada uma sobrerepresentraçăo de homens esquizofrênicos primogênitos e últimos nascidos, em nossa casuistica.

\section{DISCUSSAO}

O apoio teórico para o nosso achado pode ser objeto de discussão mas não é este o objetivo do presente estudo. Por exemplo, veja-se Raychaudhury ${ }^{31}$ ou Rollman-Branch ${ }^{32}$. Pode-se mencionar que as tensões psicológicas que pesam sobre os filhos que nascem nos extremos de suas proles têm sido enfatizadas 


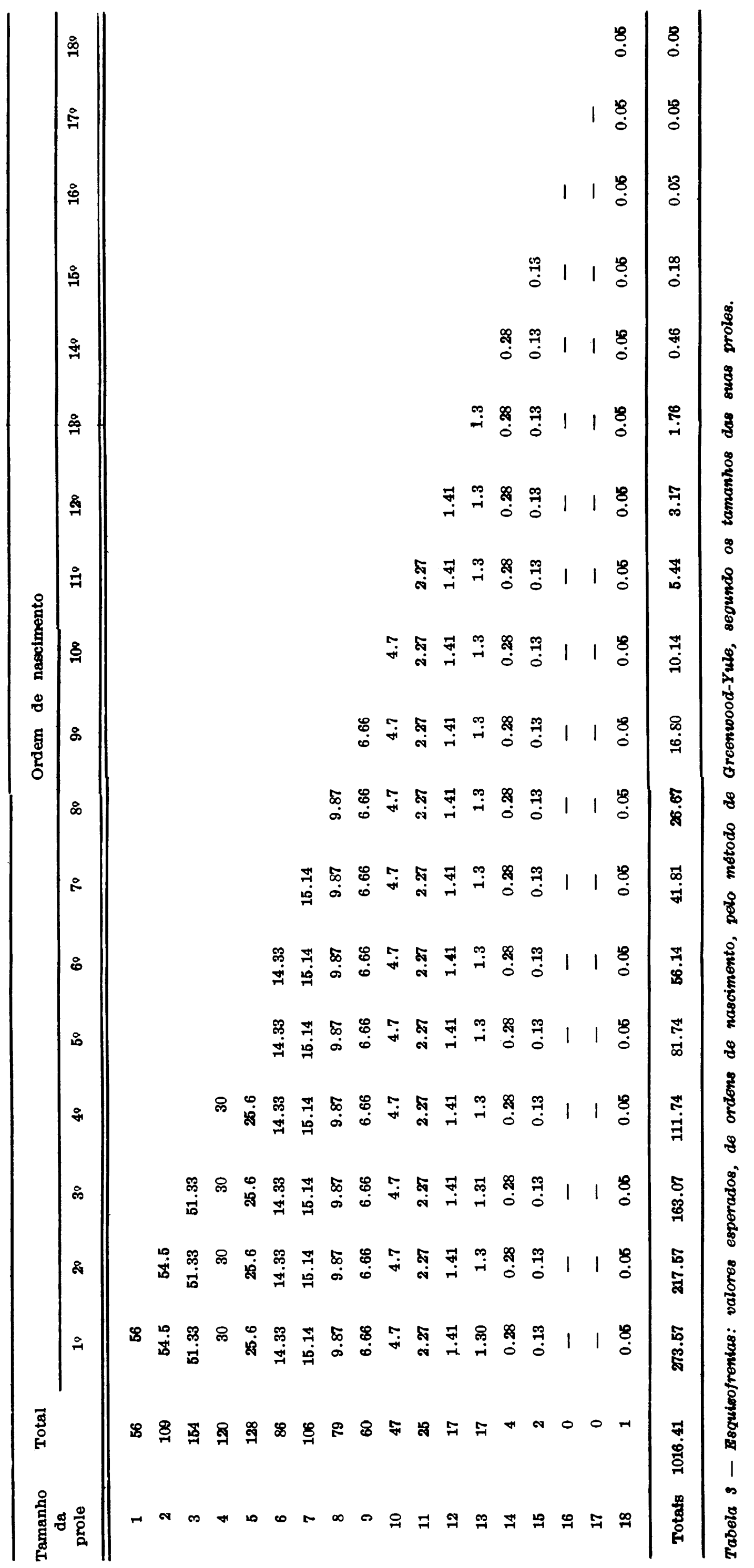




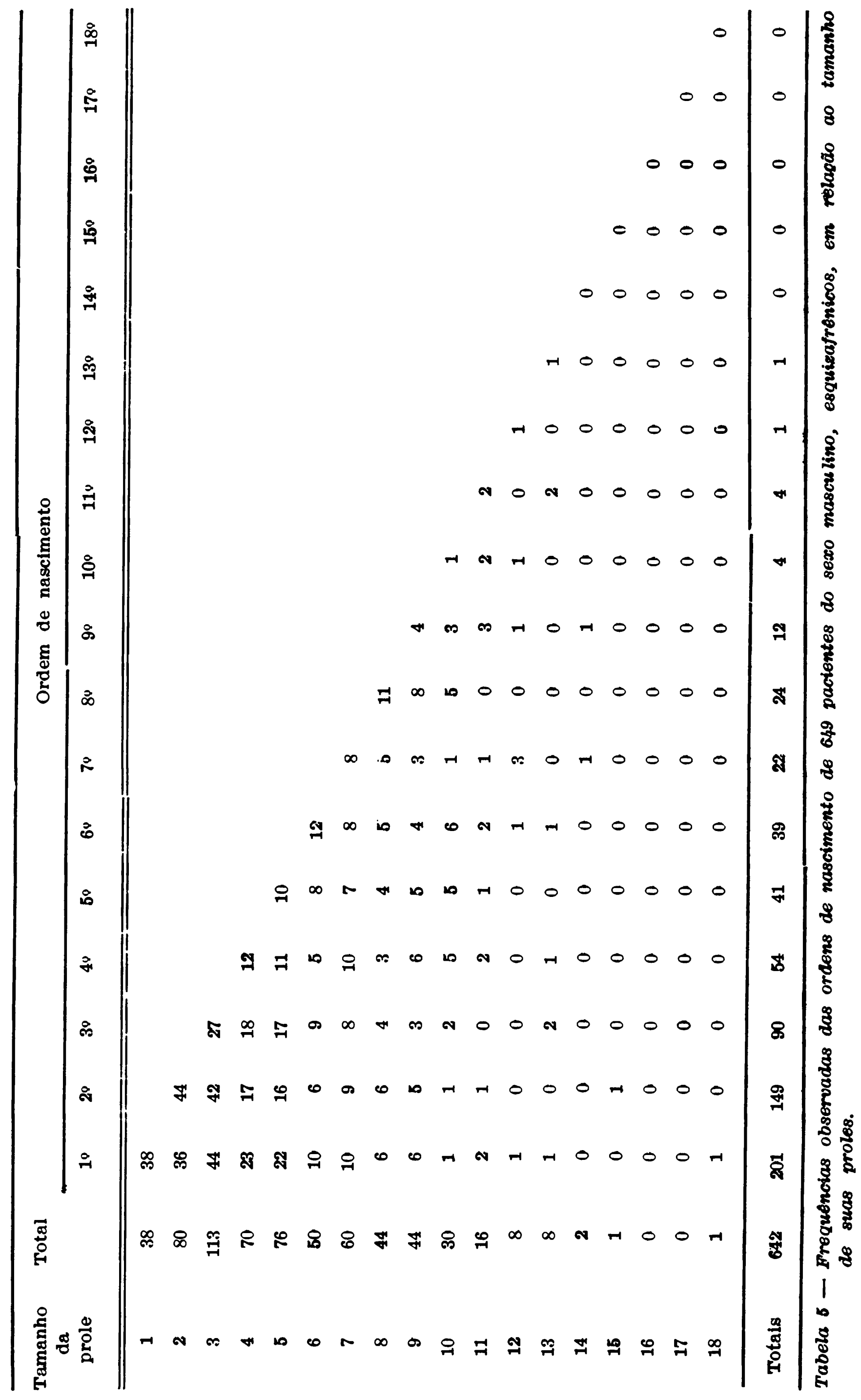




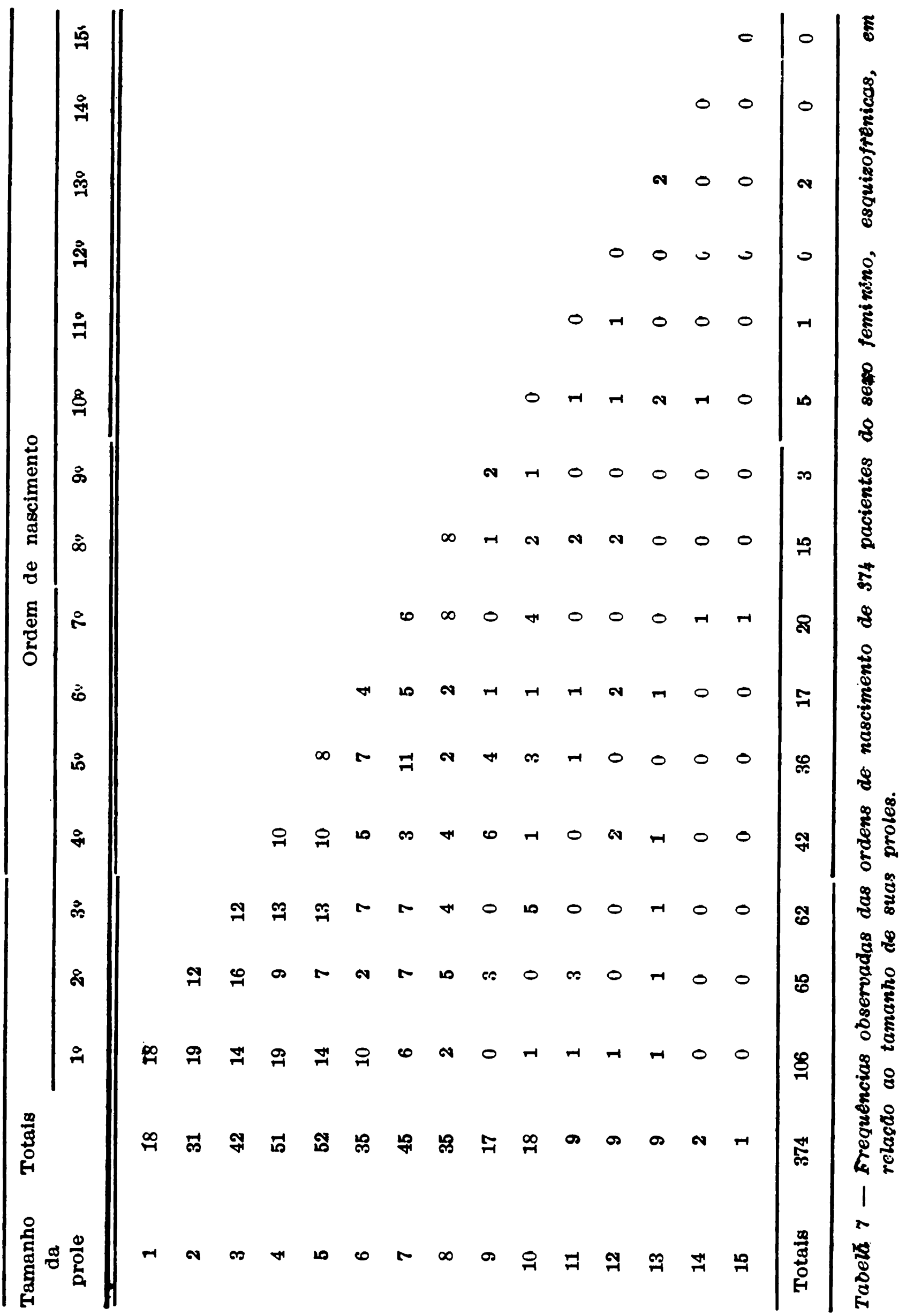




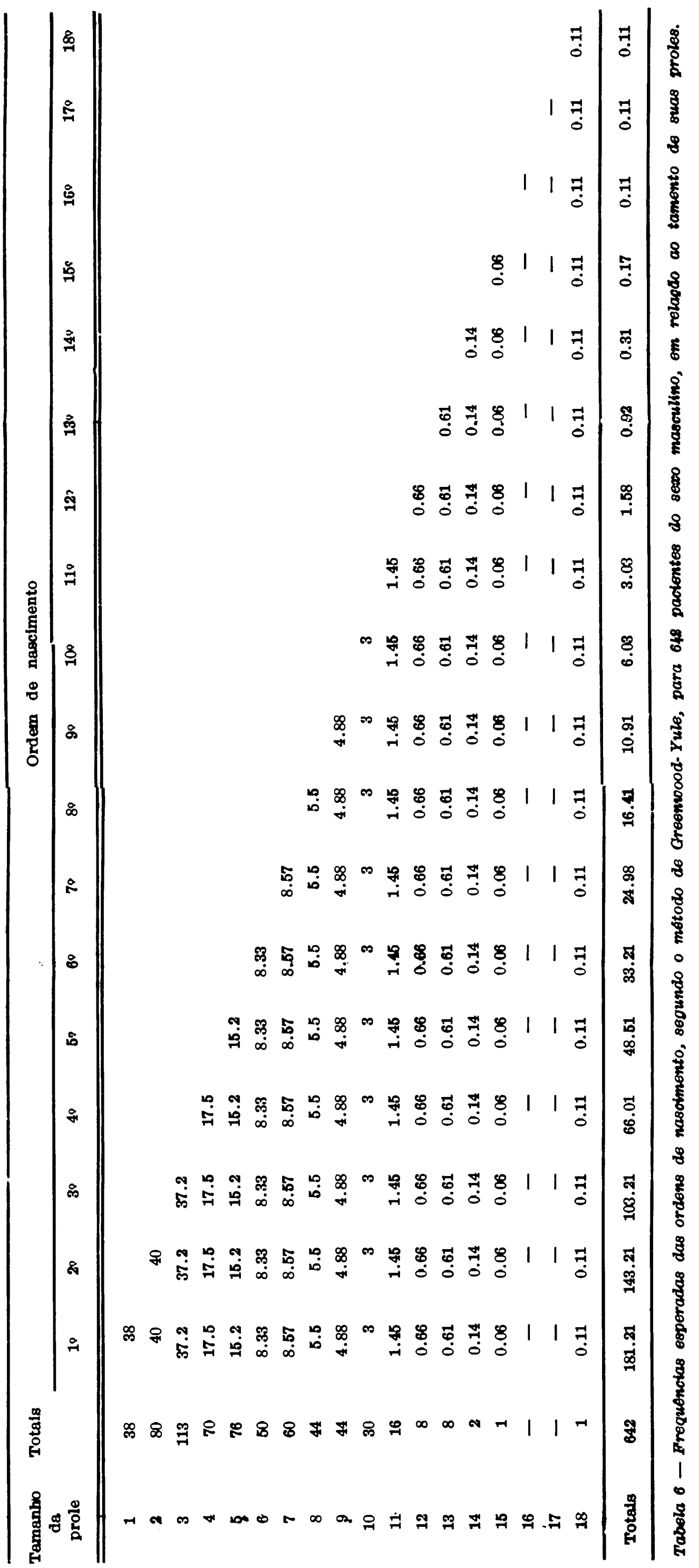




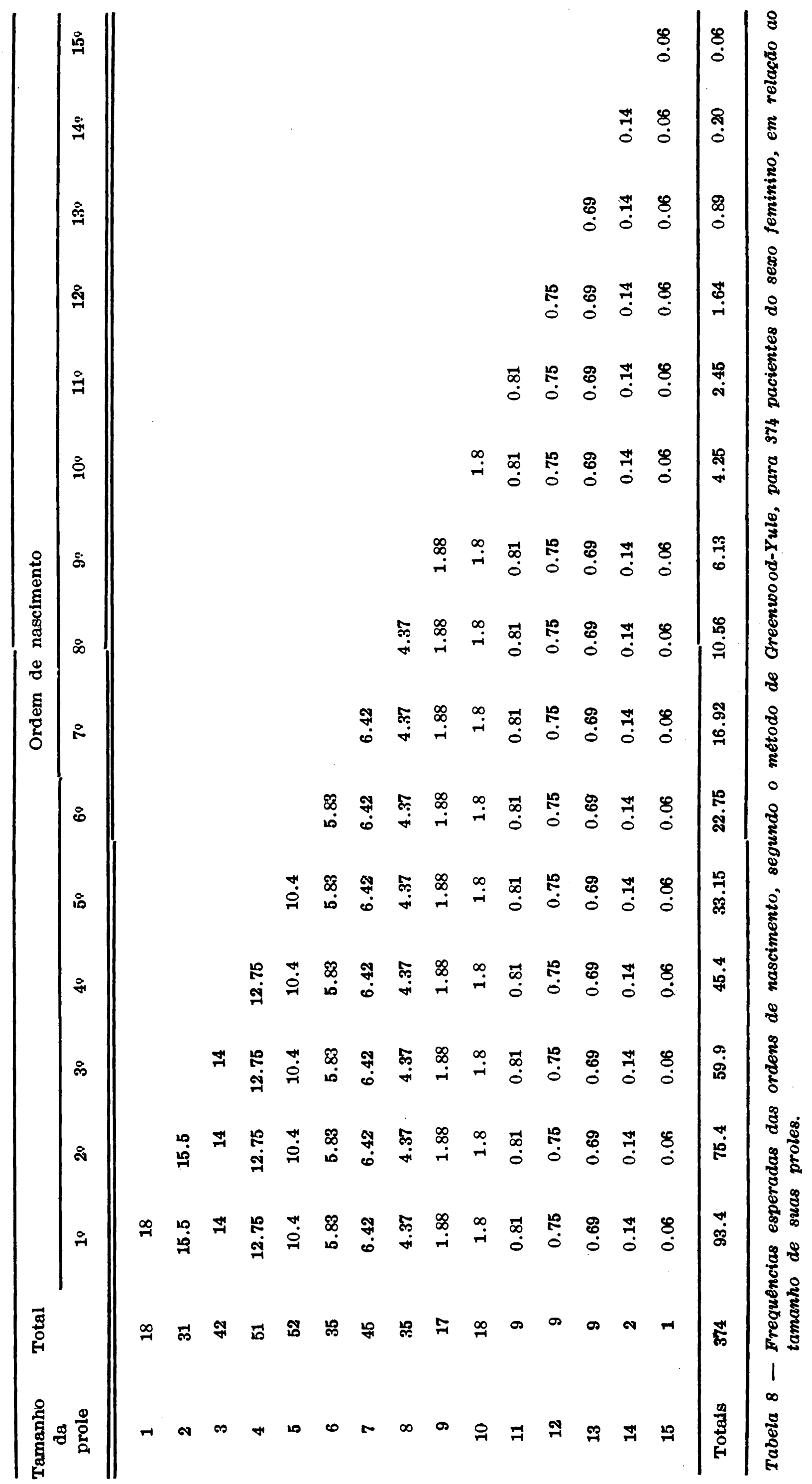




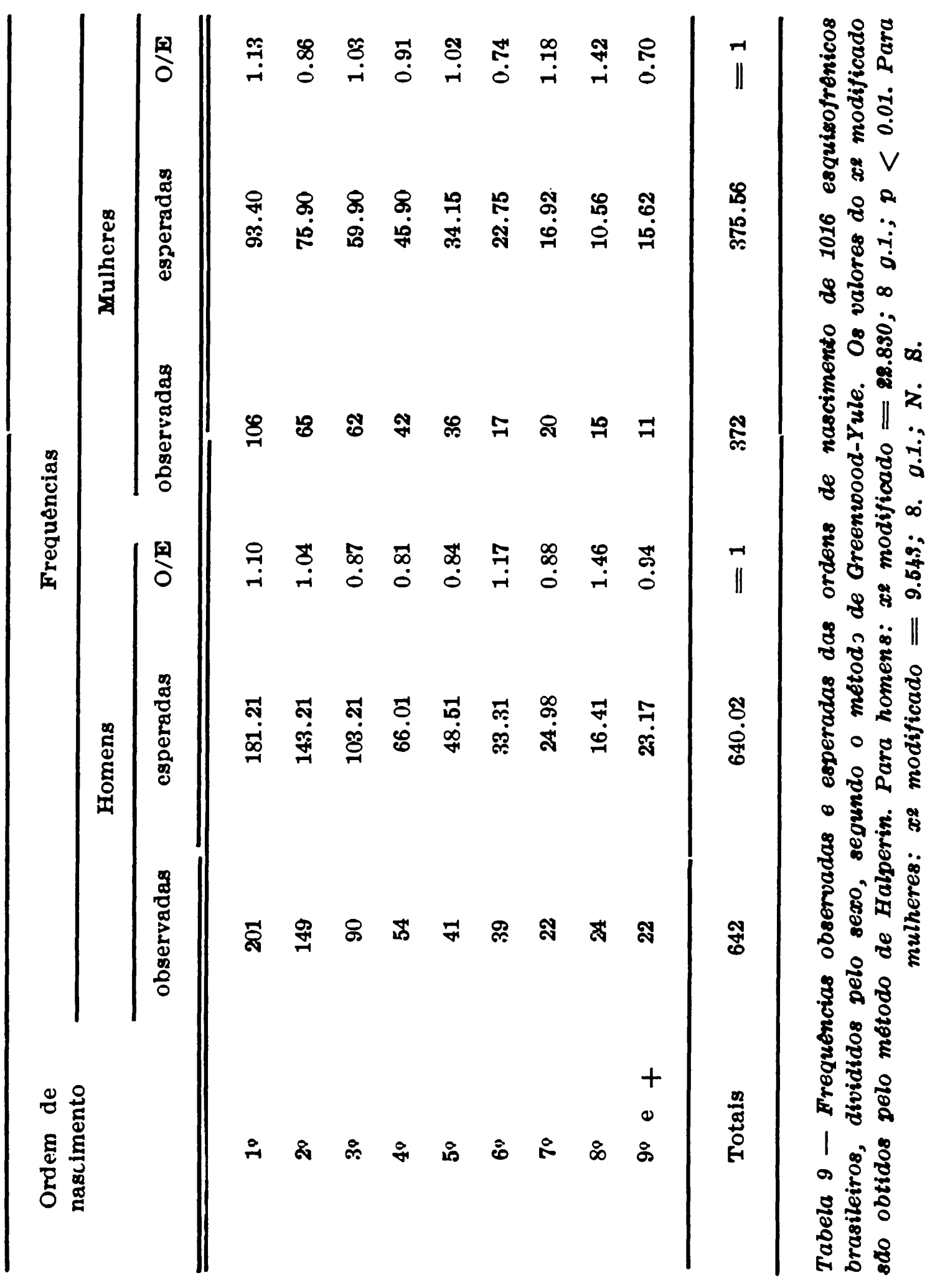


por vários autores, em várias épocas e culturas diferentes. Entre os japoneses, o primogênito é uma figura especial, o mesmo acontecendo com os caçulas indianos 43,44. Como afirmamos no início deste artigo e em outras ocasiões 34,35, acreditamos que o nosso resultado estatístico pode ser útil para a formulação mais precisa das hipóteses etiológicas a respeito da esquizofrenia, se utilizado de maneira correta. Para usar as conclusões adequadamente, uma ressalva deve ser feita: foi encontrada uma sobre-representação de homens esquizofrênicos primeiros e últimos nascidos em nossa causuística; nós não estamos afirmando que os primeiros e últimos nascidos nas proles tenham maior risco ou sejam mais propensos a desenvolver a doença. A associação não tem implicações causais diretas, em vista do fato que relações de causa e efeito diretas não são admissíveis no complexo quadro da esquizofrenia ${ }^{35}$, ou de qualquer outra doença psiquiátrica.

Breve revisão de outros estudos sobre esquizofrenia e ordem de nascimento é pertinente. Aproximadamente trinta estudos foram feitos, mas eles lançaram pouca luz sobre o problema. Mesmo em estudos comparáveis, em termos da metodologia estatística, os resultados são discordantes, e note-se que há poucos estudos realmente comparáveis, se formos rígidos no critério estatístico. Como apontamos inicialmente, alguns autores recomendaram uma parada neste tipo de investigação ?.

Não há concordância nas conclusões: aproximadamente metade dos estudos disponiveis mostraram que não existe associação estatística entre esquizofrenia $e$ ordem de nascimento $6,11,12.18,22,25,27,41,45$. A outra metade divide-se quanto às conclusões, pois alguns mostram que os primeiros nascidos aparecem mais nas casuísticas $2,7,42,43,44,47$, enquanto que outros encontraram uma sobrerepresentação dos últimos $2,7,10,15,16,38$. Um dos investigadores encontrou diferenças quanto ao sexo ${ }^{10}$, sendo que as mulheres não mostravam associação. Outros autores chegaram a conclusões discordantes dentro do mesmo estudo, ao variarem a metodologia e os dados 7 e um deles conclui curiosamente que os penúltimos nascidos (e somente eles) são mais susceptiveis de desenvolver a doença 15,16 * Esta é uma comparação grosseira dos trabalhos, e, se fizermos uma análise comparativa mais cuidadosa, segundo critérios estatisticos, perceberemos que, na verdade, os estudos não são comparáveis entre si. Eles foram conduzidos em locais e épocas muito diferentes, com métodos diagnósticos variando consideravelmente. Alguns dos autores não se utilizaram de dados próprios, aproveitando casuísticas diferentes ${ }^{7,11}$; outros, rejeitaram os gêmeos ${ }^{12}$;

* Pode-se perceber que quando o autor define a penúltima posiçăo, ele traz para a sua casuistica, homogêneamente, representantes de todas as posições de ordem de nascimento (pois o penúltimo de uma familia de três é o segundo nascido. de uma familia de quatro, o terceiro. e assim por diante) e assim ele acaba testando a năo associaçăo, e nåo uma posiçăo especifica artificialmente escolhida e definida. Esta conclusão, de Hinshelwood, provocou certa celeuma com outros autores, como Barry3. 
alguns não fizeram uso de testes de aderência ${ }^{24,47}$, enquanto que certos autores trabalharam com dados altamente específicos, de determinada classe social ou com apenas um tamanho de familia ${ }^{12,42}$.

Foi apontada a interferência das taxas de nascimento e casamento dentro de uma população, abrindo-se, como alternativa ao velho método de GreenwoodYule, um grupo controle da população. Os critérios para se escolher este grupo controle são muitos, sendo que Birtchnell* estudou o problema com este métodó.

Mesmo que estas observações sobre interferências devidas a flutuações nas características populacionais sejam válidas, existem outros fatores que podem estar desempenhando algum papel neste baixo índice de concordância. Pensamos que a confusão se origina dos subsidios metodológicos: parece-nos ${ }^{34,35}$ que os médicos não se familiarizam com os métodos estatísticos e não os usam quando eles envolvem um grau razoável de complexidade. É possível que os métodos utilizados para analizar os dados não sejam pertinentes ou confiáveis, mesmo que muitas vezes sejam fáceis de serem aplicados. $O$ método original de Greenwood-Yule usa o qui quadrado clássico, bem conhecido. Outros métodos propostos não demonstram maior potência para testar efeitos de ordem de nascimento do que o Greenwood-Yule com qui quadrado e, desta maneira, os resultados que eles propiciam não são mais confiáveis. Mas estes testes, como o de Keeping ${ }^{20}$, Slater ${ }^{40}$ e Russet-Davis ${ }^{33}$ são igualmente fáceis de serem manipulados e foram quase que imediatamente aproveitados após a sua introdução (Tsuang ${ }^{45}$, Sudaraj e Rao ${ }^{34,44}$ e Solomon e Nuttal 42). Por outro lado, o método de Max Halperin, mais difícil de ser usado, mas que matematicamente se mostra mais adequado, nunca foi aplicado, apesar de seu autor, juntamente com Mantel, ter introduzido uma simplificação em seu uso. Mesmo os autores que não se preocupam com a quantidade de trabalho envolvida, ao sugerir grupos populacionais 6,29 não se interessam pela segurança dos modelos matemáticos disponiveis.

Nossos achados são similares a aproximadamente metade dos estudos disponíveis, embora nenhum deles aponte uma tendência em direção aos primeiros e últimos nascidos, simultâneamente. Não temos a pretensão de estar com a última palavra, nem de simplificar um problema tão complexo, que envolve aspectos teóricos do domínio da macro-sociologia (principalmente traços da dinâmica intrafamiliar ${ }^{4,48}$ ) colocando-o em termos meramente estatísticos. 0 campo permanece aberto, e estudos ulteriores poderiam ser mais precisos e sofisticados, controlando e isolando outras fontes de interferência, ao levar em consideração variáveis macro-sociais e micro-sociais.

* Note-se que no Brasil, os registros oficiais de nascimento são incompletos, e as taxas populacionais de fertilidade e casamento muitas vezes inexistem. Isto torna o método de Greenwood-Yule uma escolha válida e quase obrigatória. 


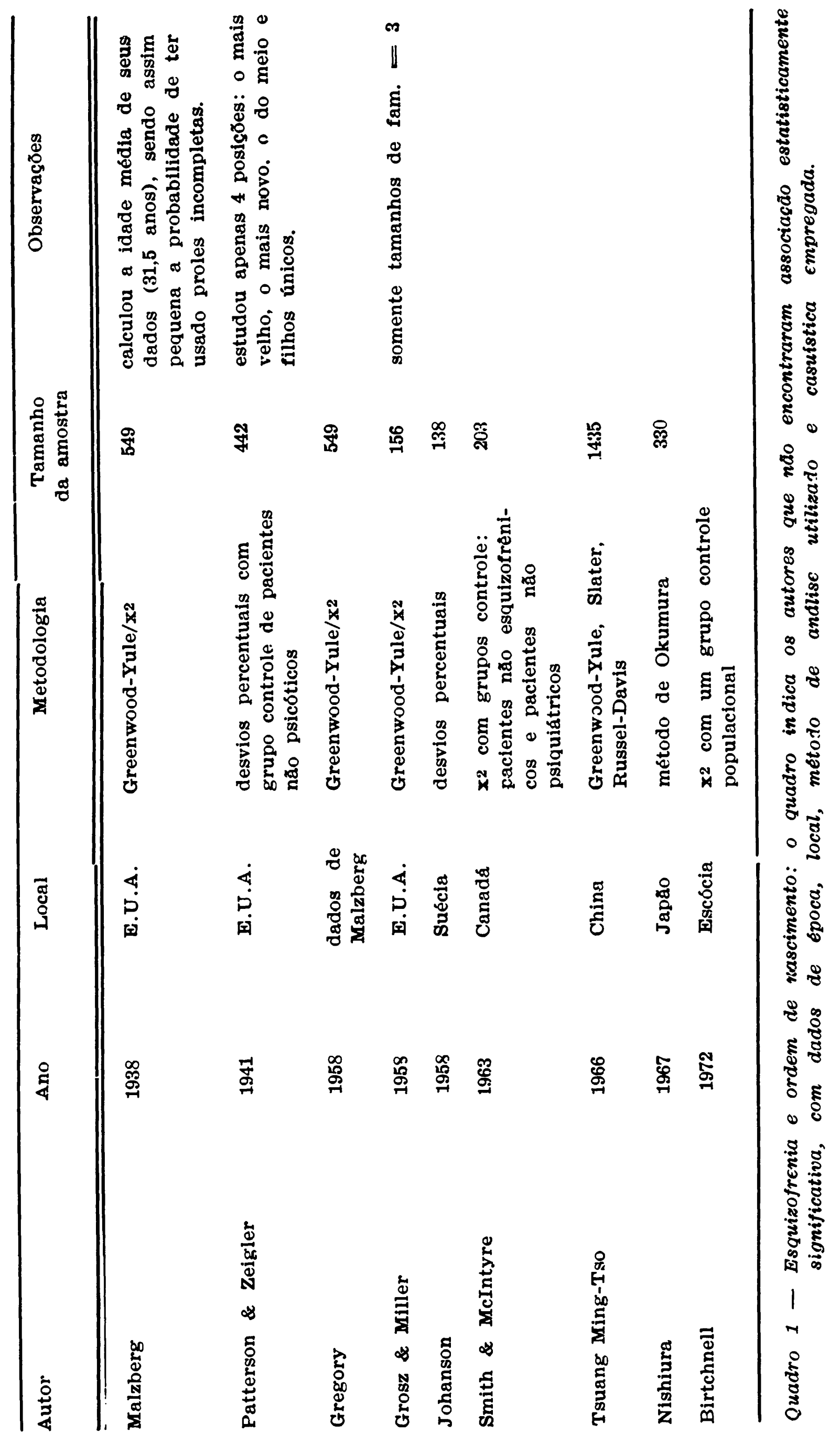




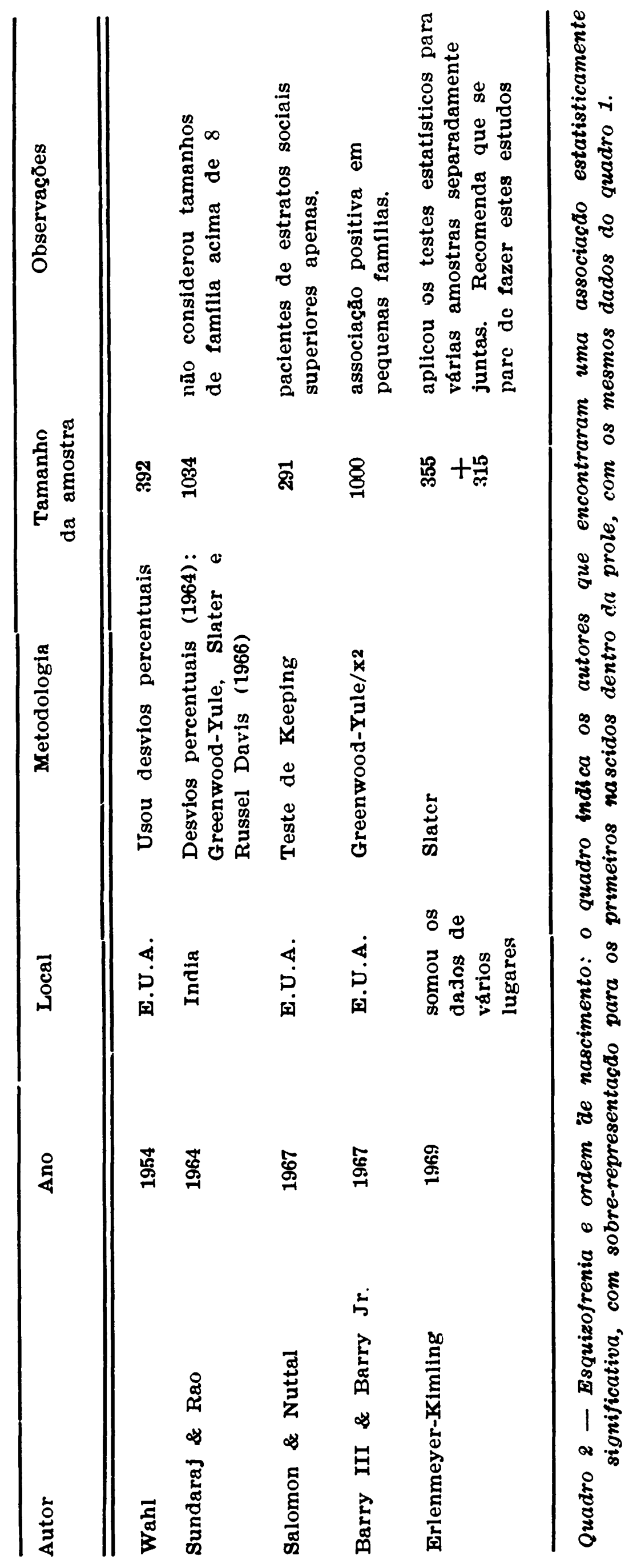




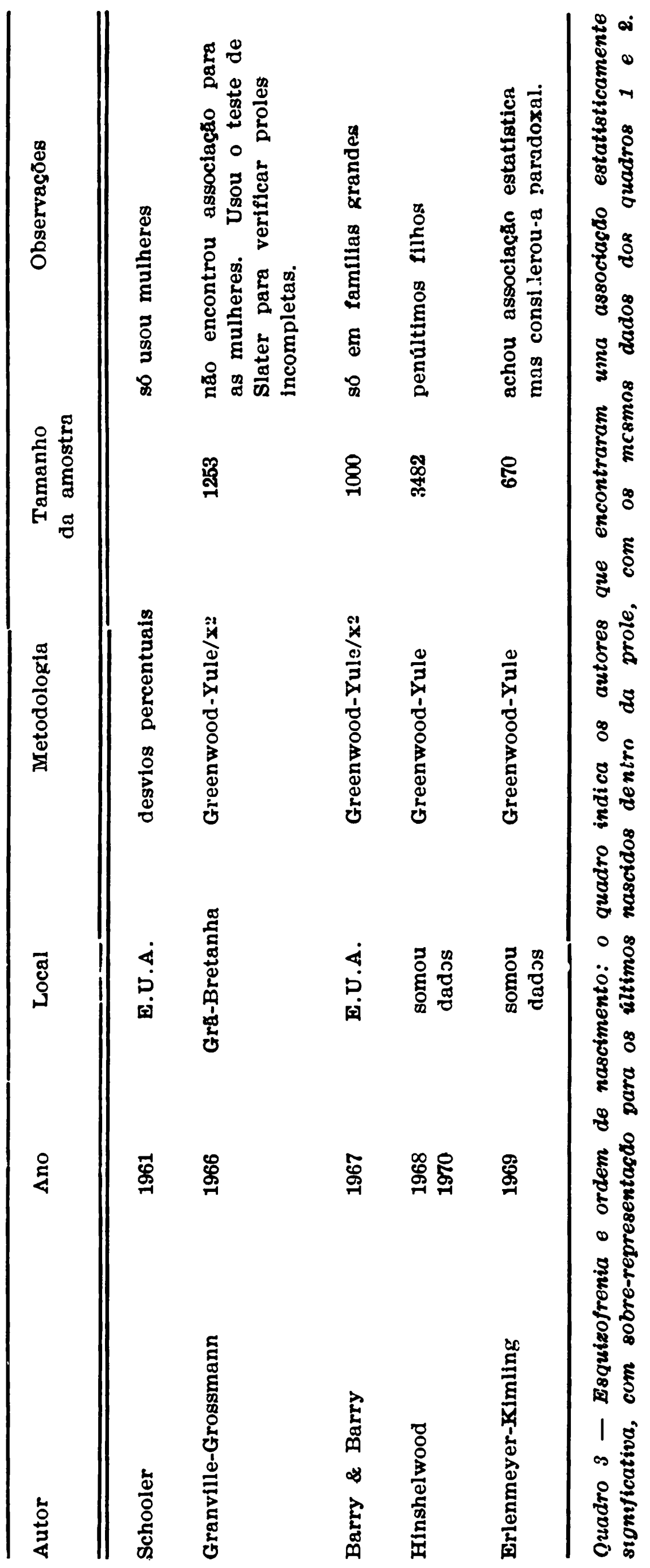




\section{RESUMO}

Mediante o possivelmente mais seguro teste de aderência para efeitos de ordem de nascimento, o qui quadrado de Max Halperin, os autores encontraram uma associação estatisticamente significante entre esquizofrenia e ordem de nascimento de 1016 brasileiros esquizofrênicos segundo padrões nosológicos da escola alemã. Esta associação foi para os homens esquizofrênicos, em direção aos primeiros e últimos nascidos em suas proles, fornecendo assim certo respaldo estatístico a teorias que enfatizam as experiências estressantes sobre crianças que estão nos extremos de suas famílias. As mulheres não mostraram qualquer associação, e a explicação para o fato pode se basear em fatores culturais. Variáveıs macro-sociais (classe sucial) e especificas do estudo (tamanho de família e proles incompletas) que poderiam interferir no estudo foram controladas. São incluídos um quadro comparativo dos dados encontrados na literatura sobre o tema e alguns comentários sobre metodologia.

\section{STIMMARY}

\section{Schizophrenia and birth order.}

With the possible most powerful goodness-of-fit test available until now, the Halperin's chi-square, a statistically significant association between schizophrenia and birth order in 1016 brazilian patients is reported. This association is with early and last born males, favoring theories which put emphasis on stressful experiences over children who are in the extremes of their sibships. There is a sex factor: females show no association. Factors which could introduce bias, as family size, social class and incomplete sibships were tentatively controlled. Since in Brazil no population data are available, the Greenwood-Yule method seems useful and valid for the purpose, with the aid provided by Halperin's chi square modification. A brief comment on results and earlier studies is made, together with suggestions for future research.

\section{REFERENCIAS}

1. BASTIDE, R. - Sociologia das Doenças Mentais. Versão brasileira. Comp. Ed. Nacional, Såo Paulo, 1967.

2. BARRY, H. Jr. \& BARRY, H. III - Birth order, family size and schizophrenia. Arch. Gen. Psychiat. $17: 435,1967$.

3. BARRY, H. Jr. - Birth order of schizophrenics. Nature 223:752, 1969.

4. BATESON, G.; JACKSON, D.; HALEY, J. \& WEAKLAND, J. - Toward a theory of schozophrenia. Behav. Sci. 1:251, 1956.

5. BLEULer, E. - Tratado de Psiquiatria. Versăo castelhana. Espasa Calpe S.A., Madrid, 1967.

6. BIRTCHNELL, J. - Birth rank and mental illness. Nature (London) 234:485. 1971.

7. ERLEMMEYER-KIMLING, L. - The problem of birth order and schizophrenia: a negative conclusion. Brit. J. Psychiat. 155:659, 1969. 
8. EY, H.; BERNARD, P. \& BRISSET, C. - Tratado de Psquiatria. Versão castelhana. Toray-Masson S. A., Barcelona, 1969.

9. FORATINI, O. P. - Epidemiologia Geral. Ed. Edgard Blücher Ltda.. São Paulon, 1976.

10. GRANVILLE-GROSSMANN, K. L. - Birth order and schizophrenia. Brit. J. Psychiat 112:1119, 1966.

11. GREGORY, I. - An analysis of familiar data on psychiatric patients: parental age, family size, birth order and orJinal position. Brit. J. Prev. Soc. Med. 12:42. 1958.

12. GROSZ, H. \& MILLER, I. - Siblings patterns in schizophrenia. Sci. 123:130, 1958.

1:3. HALPERIN. M. - The use of of $x z$ in testing effects of birth order. Ann. Eug. 18:99, 1953.

14. HARE, E. H. \& PRICE, J. S - Birth rank and schizophrenia. Nature (London) 228:1223, 1970.

15. HINSHELWOOD, R. D. - Schizophrenia and birth order: the last-but-one position. Nature (London) 220:490, 1968.

16. HINSHELWOOD, R. D. - The evidence for a birth order factor in schizophrenia. Brit. J. Psychiat. 117:293, 1970.

17. HOLLINSGSHEAD, A. \& REDLICH, F. K. - Social Class and Mental Illness. Science Ed., New York, 1964.

18. JOHANSON, E. - A study of schizophrenia in the male: a psychiatric and social study based on 138 cases with follow-up. Acta Psychiat. Neurol. Scand. 33: supl. 125, 1958.

19. KAPLAN, H. I. \& FREDMAN, A. H. - Comprehensive Textbook of Psychiatry. The William \& Wilkins Co., Baltimore, 1967

20. KEEPING, E. S. - The birth rank. Biometrics 6:112, 1952.

21. LeightoN, A.; ClAUSEN, J. A. \& WILSON. R. - Explorations in Social Psychiatry. Basic Books Inc., New York, 1957.

22. MALZBERG, B. - Is birth order related to the incilence of mental disease? Amer. J. Ph. Anthr. 24:91, 1938.

23. MANTEL, N. \& HALPERIN, M. - Analysis of birth rank data. Biometrics $19: 324$. 1963.

24. McMAHON, B.; PUGH, T. \& IPSEN, J. - Metodos de Epidemiologia. Versã، Castelhana. Prensa Medica Mexicana, Mexico. D. F.. 1965.

25. NISHIURA, H. \& TAKEUCHI, K. - Study of birth rank in schizophrenia and neurosis. Bull. Osaka Med. Sch. supl. 12:242. 1967.

26. OMS Ser. Inf. Tecn. 185. - Epidemiologia de los transtornos mentales, 1960.

27. PATTERSON, R. \& ZEIGLER, T. - Ordinal position and schizophrenia. Amer. J. Psychiat. 98.455, 1941.

28. PRICE, J. S. \& HARE, E. H. - Birth order studies: some sources of bias. Brit. J. Psychiat. 115:633, 1969.

29. PRICE, J. S. \& HARE, E. H. - Birth order studies: bias caused by changes in birth rates. Brit. J. Psychiat. 115:647. 1959.

30. RANSON, J. A. - Social Psychiatry 130:841, 1973.

31. RAYCHAUDHURY, A. K. - Are the first born more susceptible to functional mental disease? J. Nerv. Ment. Dis. 124.478. 1956.

32. ROLLMAN-BRANCH, H. - The first-born child. male: vicissitudes of pre-oedipal problems. Int. J. Psychoan. 47.404, 1966. 
33. RUSSEL-DAVIS, D. - Birth order and maternal age of homossexuals. Lancet $1: 69,1962$.

34. SANDLER, P. C. - A Psiquiatria social e a epidemiologia dos distárbios mentais. Rev. Med. São Paulo, 65:331, 1971.

35. SANDLER, P. C. - Dinamica sócio-cultural na epidemiologia da esquizofrenia. Bol. Psiquiat. 6:59, 1973.

36. SANDLer, P. C. - O papel da familia na doença mental: correlaçóes teóricas entre a psiquiatria social e antipsiquiatria. Temas (Revista do Hospital dos Servidores Públicos do Estado de Săo Paulo) 7-8:170. 1974.

37. SANDLER, P. C. - Psiquiatria social: definiçăo de termos e um esquema. Bol. Psiquiat. 8:53, 1975.

38. SCHOOLER, C. - Birth order and schizophrenia. Arch. Gen. Psychiat. 4:91, 1961.

39. SILVA, G. R. \& CASTILHO, E. A. - Métodos em Medicina Preventiva: o método epidemiológico. Publicação interna do Departamento de Medicina Preventiva da Faculdade de Medicina da Universidade de são Paulo, 1969 (mimeografado).

40. SLATER, E. - Birth order and maternal age of homossexuals. Lancet, i:540, 1962.

41. SMITH, D. \& McINTYRE, A. - Family size, birth rank and ordinal position in psychiatric illness. Canad. Psychiat. Ass. J. 8:244, 1963.

42. SOLOMON, L. \& NUTTAL, R. - Sibling order, premorbid adustment and remission in schizophrenia. J. Nerv. Ment. Dis. 144:37, 1967.

43. SUNDARAJ, N. \& RAO, B. - Birth order and schizophrenia. J. Nerv. Ment. Dis. 138:87, 1964 .

44. SUNDARAJ, N. \& RAO, B. - Order of birth and schizophrenia. Brit. J. Psychiaty 112:1127, 1966.

45. TSUANG, MING-TSO - Birth order and maternal age of psychiatric-in-patients. Brit. J. Psychiat. 112:1131, 1966.

46. VERóN, E. - Perspectivas futuras en la investigación básica sócio-cultural sobre salud mental. Acta Psiquiat. Psicol. Amer. Lat. 193:210, 1968.

47. WAHL, C. W. - Some antecedent factors in the family histories of 392 schizophrenics. Amer. J. P. Psychiat. 110:668, 1954.

48. WYNNE, L.; RYCKOFF, I.; DAY, J. \& HIRSCH, S. - Pseudo-mutuality in the family relations of schizophrenia. Psychiat. 21:205, 1958.

49. zUBIN, J. \& FREYhaN, A. - Social Psychiatry. Grunne \& Stratton, New York, 1968.

Instituto AchE - Avenida Lacerda I'ranco 587 - 01596 Sä Paulo, SP - Brasil 